\title{
Carnets
}

Revue électronique d'études françaises de l'APEF

Deuxième série - $18 \mid 2020$

Chiens et écritures (littéraires, filmiques, photographiques)

\section{Les chiens dans Los Pazos de Ulloa, et les inflexions d'un roman naturaliste}

\section{Yves Germain}

\section{(2) OpenEdition}

\section{Journals}

Édition électronique

URL : http://journals.openedition.org/carnets/10741

DOI : $10.4000 /$ carnets. 10741

ISSN : 1646-7698

Éditeur

APEF

Référence électronique

Yves Germain, «Les chiens dans Los Pazos de Ulloa, et les inflexions d'un roman naturaliste ", Carnets

[En ligne], Deuxième série - 18 | 2020, mis en ligne le 31 janvier 2020, consulté le 04 juin 2020. URL:

http://journals.openedition.org/carnets/10741 ; DOI : https://doi.org/10.4000/carnets.10741

Ce document a été généré automatiquement le 4 juin 2020

\section{(c) (i) \&}

Carnets est mis à disposition selon les termes de la licence Creative Commons - Atribution - Pas d'utilisation commerciale 4.0 International. 


\title{
Les chiens dans Los Pazos de Ulloa, et les inflexions d'un roman naturaliste
}

\author{
Yves Germain
}

1 Bien souvent, l'animal n'a dans les textes qui ne le mettent pas spécifiquement en scène, que le statut d'un détail, semblable alors à celui des petits animaux qui, dans les portraits aristocratiques de l'âge classique, semblent un ornement parmi tant d'autres (Laneyrie-Dagen, 2016). Le détail pourtant, comme l'a si bien montré Daniel Arasse dans ses études iconographiques, peut dépasser cette fonction secondaire d'ornement ou d'anecdote, pour dire ou révéler bien plus que ce qu'on lui conférait comme valeur initiale, « comme une expérience qui n'est secondaire qu'en apparence » (Arasse, 1996: 6). C'est en pensant à ce statut apparemment marginal et profondément significatif, que nous nous proposons d'examiner la place des chiens dans un roman d'une romancière espagnole qui n'a rien d'un auteur animalier, Emilia Pardo Bazán dans Los Pazos de Ulloa. Ce roman, le cinquième de la romancière galicienne, qui, sous l'égide de Galdós, s'est déjà essayée au naturalisme avec l'évocation des ouvrières de la fabrique de cigares de La Corogne, La Tribuna (1883), inaugure en 1886 un diptyque consacré à la ruralité galicienne, qui se poursuivra l'année suivante avec La madre naturaleza, formant ainsi un ensemble tenu à juste titre comme un aboutissement de sa période naturaliste. Or doña Emilia, peu attentive en général à la matière animalière, fait avec ce premier roman rural une exception notable, où la présence des chiens s'impose dans la logique de l'évocation de la petite noblesse galicienne, isolée dans ses manoirs ou pazos où la chasse est l'activité essentielle d'une caste de hobereaux décadents, vivant dans la promiscuité avec leurs domestiques. Pour la romancière, montrer l'omniprésence des chiens contribue à signaler cet abaissement dans la ruralité qui indigne le nouveau chapelain des Pazos, Julián. À ce titre, la thématique canine et cynégétique est d'abord une contribution à la mise en place d'un "état du milieu», au sens zolien, une production de signes rendant compte d'un mode de vie où le hobereau chasseur fait pour ainsi dire corps avec ses chiens. La logique du projet littéraire semble induire la 
présence canine, en renforcement d'autres caractéristiques du comportement humain, telles que la promiscuité sexuelle du maître et de la servante.

2 Toutefois, ce roman ne saurait se lire comme la seule illustration d'un naturalisme d'école - Pardo Bazán avait pris soin, au demeurant, de se démarquer nettement, dans ses essais critiques de La cuestión palpitante, d'une approche trop calquée sur une école française qui dérangeait sa posture d'auteur catholique par la part excessive accordée au déterminisme du milieu - ; Los Pazos est aussi un roman plus divers, qui intègre une part de l'imaginaire du roman gothique et explore aussi les inquiétudes de l'âme humaine, à travers la figure du chapelain timoré qui ne parvient pas à s'avouer qu'il aime Nucha, son amie d'enfance et l'épouse fragile qu'il a fait épouser au marquis d'Ulloa pour tenter de soustraire celui-ci à sa vie dans le péché avec la servante Sabel. Or précisément, la matière animale, convoquée initialement pour servir l'état du milieu naturaliste, s'avère plus fertile et dépasse sa fonction illustrative pour accompagner les mouvements de l'imaginaire de la romancière, sollicité par l'atmosphère trouble de ce " pays de loups » qui entoure le manoir.

Qu'on ne s'y trompe pas : ce roman de mœurs et de psychologie est assurément loin d'être un roman à matière animale. Tout comme dans ses contes, Pardo Bazán reste éloignée de cette thématique telle qu'on peut la trouver chez Maupassant, ou dans certains contes de Clarín qu'il peut être intéressant de convoquer en comparaison pour évaluer la présence de la thématique animale dans le naturalisme espagnol : seul clarín, apparemment, manifeste une réelle empathie avec la gent canine, notamment. Son approche des chiens, toutefois, est avant tout anthropocentrique et reproduit, en fin de compte, une bonne part de l'héritage cervantin du Colloque des chiens où l'animal reflétait l'homme, pour dénoncer ses travers et son ingratitude. L'introduction des chiens dans le monde rural galicien de Pardo Bazán ignore cette assimilation anthropocentrée: l'animal n'y apparaît que comme tel, une présence associée à l'environnement, une composante du milieu. Sa présence reste de l'ordre du détail, et seul le questionnement du présent colloque nous incite à l'examiner de plus près. Et pourtant ces quelques détails ne manquent pas d'éclairer avec force plusieurs dimensions significatives du roman.

4 L'animal en général et le chien en particulier ne semblent pas avoir suscité chez doña Emilia une véritable empathie. On ne retrouve guère en Espagne à l'époque la sensibilité à l'animal, que l'on peut rencontrer par exemple chez Maupassant (1974), avec l'histoire du cocher François forcé à noyer sa chienne Cocotte, qui lui inspire coup sur coup deux contes, «Histoire d'un chien» (1881), plaidoyer explicite pour la Société protectrice des animaux (fondée en 1845), puis « Mademoiselle Cocotte » (1883), où le bourreau malgré lui de la chienne devient fou... Seul Clarín, à bien des égards le rival de Pardo Bazán, paraît s'en approcher. Comme elle critique et romancier, prétendant autant qu'elle au rôle d'introducteur du naturalisme, il devient un des plus sévères critiques de la romancière à partir de 1888 et de la publication d'Insolación. Chez lui, justement, le motif du chien est récurrent, avec l'histoire d'un perro de lanas, un grand caniche, qu'il reprend après un premier essai dans «El Quin » (1895), intégré l'année suivante dans les Cuentos morales, le récit cruel d'un abandon par le maître que ce chien s'était en quelque sorte choisi (Clarín, 2012: $275 \mathrm{sq}$ ). Mais si le chien est là le cœur de l'histoire (" en ella se trata de estudiar el estado de alma de un perro »), la facture du conte peut paraître assez traditionnelle. Le Berganza du Coloquio de los perros cervantin ne racontait pas autrement sa vie soumise à l'ingratitude de ses maîtres successifs. 
Clarín se contente de rajouter quelques notations d'époque, ou le rêve romantique d'un chien aspirant à des amours bucoliques, et vite déçu par la polygamie vulgaire des chiennes de la campagne. De même, l'ultime déception du «Quin », qui recherche une consolation dernière à son abandon en se réfugiant dans une église, où on le reçoit, comme le dit l'espagnol, como perro(s) en misa (comme un chien dans un jeu de quilles, en français) fait-elle écho aux inquiétudes spirituelles de Clarín lui-même. Placer l'animal au centre du récit ne permet pas alors de le soustraire à un anthropocentrisme fondamental, où le chien demeure le miroir des injustices et de l'ingratitude, dont il est victime à l'image des hommes. Ce bref détour par l'amour des chiens de Clarín entend aider à mieux saisir l'apport bien différent de Pardo Bazán à la représentation des chiens et à leur intégration cette fois dans un projet romanesque, dans une véritable optique naturaliste, non comme sujets mais comme objets signifiants, au sein d'une ruralité présentée comme primitive.

Dès les premières pages du roman, lorsque le futur chapelain Julián s'enquiert sur la route de la distance qui lui reste à parcourir jusqu'au manoir d'Ulloa, il s'entend répondre « la carrerita de un can » (Pardo Bazán, 1997 : 96), le parcours d'un chien... La phrase, censée être en "dialecto", comme Pardo Bazán désigne le galicien, mais formulée en castillan dans le texte, situe d'emblée, en associant le chien à la mesure du monde, l'importance qui est la sienne dans cet univers de chasseurs (Julián va d'ailleurs bientôt croiser la route du marquis et de ses "canes perdigueros »). La formule, «la carrerita de un can ", s'entend bien sûr comme expression typique du caractère des Galiciens, de leur propension à dissimuler leurs intentions et à cultiver un certain mystère, mais elle confère également à l'animal un statut emblématique, de possible gardien des secrets du lieu. Et Julián ne tarde pas, en contemplant la nature austère et lugubre qui l'entoure, à glisser des chiens aux loups et à se dire " i qué país de lobos ! ».

La scène clé qui occupe le début du chapitre 2, lorsque le chapelain arrive au manoir, nous situe bien du côté des chiens et de leur présence centrale dans le monde domestique représenté par la cuisine. Le marquis prend soin qu'ils soient nourris en priorité. C'est alors que Julián s'aperçoit qu'au milieu des chiens affamés rampe un enfant, qui ne tarde pas à s'attirer des coups de dents, malgré l'évidente proximité qui le lie aux bêtes.

Julián creyó al pronto que se había aumentado el número de canes, tres antes y cuatro ahora ; pero al entrar el grupo cánino en el círculo de viva luz que proyetaba el fuego, advirtió que lo que tomaba por otro perro no era sino un rapazuelo de tres a cuatro años, cuyo vestido, compuesto de chaquetón acastañado y calzones de blanca estopa, podía desde lejos equivocarse con la piel bicolor de los perdigueros, con quienes parecía vivir el chiquillo en la mayor inteligencia y más estrecha fraternidad. (Pardo Bazán, 1997 : 106)

7 Le terme qui souligne leur promiscuité, "estrecha fraternidad», cette notion de fraternité des êtres naturels du lieu, pourrait fonctionner comme une prolepse cachée de l'intrigue à venir : le petit s'avèrera être le fils naturel du marquis et de la servante, appelé dans le roman suivant à développer un amour incestueux avec sa demi-sœur. Mais pour l'instant, c'est le scandale visible de la promiscuité avec la meute, la confusion des espèces, et l'indifférence du marquis aux pleurs de l'enfant à peine mordu, qui suscite l'indignation de Julián dans une scène longuement détaillée de son point de vue. Un scandale aussitôt accru par le fait que l'assistance invite alors l'enfant à boire du vin pour oublier son chagrin. La scène doublement scandaleuse - et Julián ne sait pas encore qui est le père de l'enfant - marque de toute évidence l'irruption dans le 
roman d'une thématique naturaliste d'autant plus susceptible de choquer qu'elle implique la petite enfance, habituellement objet en Espagne de toutes les mièvreries : la promiscuité avec les chiens, dans la saleté de la salle commune, le rapport indifférencié à l'animal et à l'enfant, l'éducation aberrante et le motif de l'alcool, tout contribue au tableau destiné à choquer les esprits et à souligner l'arriération des occupants du lieu, d'autant plus que l'interprète de la scène est ce jeune ecclésiastique dont le roman ne tarde pas à souligner l'hypersensibilité presque maladive.

Pour une romancière qui, à peine trois ans plus tôt (La cuestión palpitante, 1883), introduisait le naturalisme français auprès des lecteurs espagnols en exprimant ses réserves envers ses excès et la notion inacceptable à ses yeux d'un déterminisme du milieu, la conversion à la nouvelle école littéraire est bien là, tant par la volonté de choquer que par l'illustration d'un déterminisme de fait que cette ruralité fait peser sur ses habitants. «La aldea, cuando se cría uno en ella y no sale de allí jamás, envilece, empobrece y embrutece ", seront les paroles dont se souviendra Julián au terme du chapitre, un jugement de l'oncle du marquis qui justifiait là l'entreprise de rédemption à mener par le jeune chapelain.

La suite du roman revient à plusieurs reprises - sur le mode du détail dans des passages descriptifs ou narratifs - sur l'interaction de l'homme et des chiens, ou des chasseurs et de leurs compagnons canins. Le marquis d'Ulloa, chasseur passionné, est un homme aux chiens, inséparable de ceux-ci, comme lorsqu'il apparait à la fête de la Rectorale, au chapitre 6: "entraba seguido de dos perros perdigueros, cuyos cascabeles acompañaban su aparición con jubiloso repique »; ils complètent alors par leur présence la sensation d'un tableau de l'école flamande («apoyando la cabeza en todas las rodillas, lamiendo aquí un plato y zampándose un bizcocho allá »). Une présence canine identifie aussi, de façon pour ainsi dire emblématique, la figure plus archaïque du señorito de Limioso, autre hobereau de la région auquel les Ulloa rendent visite dans son château en ruines: l'interaction du milieu et de l'animal opère alors un effet mimétique sur le vieux chien de garde, qui tient à peine debout.

Estaba el portón abierto de par en par (...) al sonido mate de los cascos de las monturas en el piso herboso del patio respondieron asmáticos ladridos y un mastín y dos perdigueros se abalanzaron contra los visitantes, desperdiciendo por las fauces el poco brío que les quedaba, pues ninguno de aquellos bichos tenía más que un erizado pelaje sobre una armazón de huesos prontos a agujerearlo al menor descuido. El mastín no podía, literalmente, ejecutar el esfuerzo del ladrido: temblábanle las patas, y la lengua se salía de un palmo entre los dientes, amarillos y roídos por la edad. Apaciguáronse los perdigueros a la voz del señor de Ulloa, con quien habían cazado mil veces; no así el mastín, resuelto sin duda a morir en la demanda... (Pardo Bazán, 1997 : 251)

10 Les chiens sont ici à l'image d'une fin de race, d'une catégorie de petite noblesse en extinction. Le clergé rural traditionnel n'échappe pas à cette interaction liée à la manie cynégétique: ainsi Julián retrouve-t-il, dans la chambre qu'il hérite de son prédécesseur, entre autres pièges à perdrix, des colliers de chiens et une peau de lapin mal séchée et sentant bien mauvais» («collares de perros, una piel de conejo mal curtida y peor oliente»), que le jeune chapelain qualifie de reliquias/reliques, comme les restes d'un culte dévoyé.

11 Le roman demeure en revanche plus timide que la longue nouvelle qui lui avait servi d'ébauche, Bucólica (publiée dans La Revista de España en 1884), en ce qui concerne la mise en évidence d'une forme d'animalité des personnages de paysans. Le narrateur en 
est un Madrilène un peu dépassé par la brutalité des mœurs des métayers de ses parents. "Así viven, como bestias en el establo ", observe-t-il, et à plusieurs reprises, il perçoit la fille de ferme comme " un perro manso ", un chien docile qui l'attendrit. Il ne tarde pas à fauter avec elle et se croit alors obligé de l'épouser avant de réaliser que l'on abuse de sa naïveté. Certes, dans le roman, le marquis d'Ulloa traite aussi la servante de chienne lorsqu'il apprend qu'elle le trompe avec un journalier, mais cette perspective plus crue reste limitée à une scène violente du chapitre 7 ; la perspective narrative dominante, qui adopte la focalisation sur Julián, le chapelain, restreint ce regard condescendant sur les personnages populaires en même temps qu'elle limite toute référence directe à la sexualité en général.

Dans Los pazos, néanmoins, les références à l'animalité et au monde animal ne se limitent pas à l'évocation du milieu social et à l'optique naturaliste. Le manoir devient aussi peu à peu l'espace d'un imaginaire inquiétant pour les deux nouveaux venus, Julián et Nucha, la jeune épouse du marquis, tous deux dominés par une sensibilité exacerbée, et même profondément morbide dans le cas de Nucha. La perception initiale d'un "país de lobos » est reflétée assez tôt dans le blason des Ulloa, avec deux loups rampants que seul un œil avisé saurait percevoir dans la végétation touffue de buis du jardin, lointain souvenir d'un parterre du siècle précédent où l'art topiaire les y avait inscrits: " las armas de la casa eran ahora intrincado matorral de bojes donde ni la vista más lince distinguiría rastro de los lobos, pinos, torres almenadas, roeles y otros emblemas que campeaban en el preclaro blasón de los Ulloa ». Ce paradigme des loups cachés va devenir l'autre versant des chiens bien réels de la cuisine et des parties de chasse, celui d'une bestialité effrayante à même de se manifester dans l'environnement d'un roman gothique. De retour au manoir après avoir arrangé à Santiago le mariage du Marquis, Julián éprouve à nouveau la sensation d'un « triste país de lobos » à l'approche du manoir. La présence ensuite de Nucha qu'il croit menacée développe peu à peu ces appréhensions, accentuées par l'assemblée trouble qui entoure une Sabel toujours présente ; il s'alerte au moindre bruit nocturne, cri animal ou gémissement humain :

Creyó escuchar de nuevo el ay tristísimo. ¿ Serán los perros ? Asomóse a la ventana : la luna bogaba en un cielo nebuloso, y allá a lo lejos se oía el aullar de un perro, ese aullar lúgubre que los aldeanos llaman ventar la muerte, y juzgan anuncio seguro del próximo fallecimiento de una persona. Julián cerró la ventana estremeciéndose. (Pardo Bazán, 1997 : 295)

L'imaginaire galicien et son monde de sorcellerie et de prédictions est de la partie. Le hurlement sinistre n'est-il pas après tout inscrit dans le toponyme Ulloa, certes bien attesté en Galice, mais qui connote aussi ce cri? Les armes d'Ulloa réapparaissent pour prendre vie dans un cauchemar du chapelain, où le manoir du XVIII ${ }^{\mathrm{e}}$ se transforme en château féodal (Nelly Clemessy avait justement traduit le titre du roman Le château d'Ulloa) ; l'emblème demeure mais les loups s'animent et font entendre leur hurlement :

Lo único que en el Castillo recordaba los Pazos actuales, era el majestuoso escudo de armas : pero aun en este mismo existía diferencia notable, pues Julián distinguía claramente que se habían animado los emblemas de piedra, (...) y los dos lobos rampantes movían la cabeza exhalando aullidos lúgubres. (Pardo Bazán, 1997 : 297).

C'est plus encore la fragile Nucha qui va ressentir avec une angoisse incontrôlable ces appréhensions, inspirées du roman gothique ou, avant, des nouvelles de María de Zayas, au XVII siècle, une lecture que Pardo Bazán mentionne à plusieurs reprises et où elle a pu lire l'histoire d'une épouse légitime traitée en esclave, mangeant sous la table la pitance des chiens (cf. Zayas, 1998: 236-237). Pour surmonter sa peur, la jeune 
femme, au chapitre 20, convainc Julián de lui faire visiter les recoins et caves du manoir, le cloître où un anneau rouillé rappelle la souffrance d'un esclave, puis le soussol où ils descendent tandis que l'orage gronde. Nucha sursaute alors avec effroi, se croyant surprise par un énorme chien, qui n'existe que dans son imagination. " $i$ Qué sucede, señorita querida, qué sucede ? gritó el capellán. - Nada... Nada ! tartamudeó la señora de Ulloa. Se me figuró al abrir que estaba ahí dentro un perro muy grande, sentado, y que se levantaba y se me echaba encima para morderme... " (Pardo Bazán, 1997 : 305). La visite, marquée par d'autres impressions fantasmagoriques, s'achève par un évanouissement.

15 Une autre fantasmagorie, reliée non plus aux chiens mais toujours à l'imaginaire cynégétique, intervient peu après, au terme du chapitre 22, en mettant en œuvre une écriture nettement plus originale. Les deux chapitres qui succèdent à l'exploration mouvementée du manoir s'offrent comme une pause plutôt plaisante, nourrie d'abord de récits de chasseurs puis d'une séquence où Julián, contraint à prendre part à une sortie de chasseurs, fait piètre figure et déçoit son associé canin par sa maladresse à chasser la perdrix. La fin du chapitre est consacrée à l'attente des lièvres par le même chasseur inexpérimenté : mais l'écriture change radicalement, devenant un vrai poème en prose où "le chasseur", sans nom cette fois, devient le spectateur d'une parade nuptiale où l'animal - lièvres mâles et femelle - accèdent au rang de sujets d'une tragédie où la mort doit succéder à l'amour.

A la claridad lunar divisa por fin un monstruo de fantástico aspecto, pegando brincos prodigiosos, apareciendo y desapareciendo como una visión : la alternativa de la oscuridad de los árboles y de los rayos espectrales y oblicuos de la luna hace parecer enorme a la inofensiva liebre, agiganta sus orejas, presta a sus saltos algo de funambulesco y temeroso, a sus rápidos movimientos una velocidad que deslumbra. Pero el cazador, con el dedo ya en el gatillo, se contiene y no dispara. Sabe que el fantasma que acaba de cruzar al alcance de sus perdigones, es la hembra, la Dulcinea perseguida y requestada por innumerables galanes en la época del celo, a quien el pudor obliga a ocultarse de día en su gazapera, que sale de noche, hambrienta y cansada, a descabezar cogollos de pino, y tras de la cual, desalados y hechos almíbar, corren por lo menos tres o cuatro machos, deseosos de románticas aventuras. (...) (Pardo Bazán, 1997 : 318)

L'intérêt du passage est souligné par une association explicite du lièvre femelle et de Dulcinée, renvoyant à l'ultime illusion de Don Quichotte lorsqu'il rentre dans son village et croise la route d'un lièvre. Le récit, signalé aussi par le jeu des temps - l'usage, déconcertant dans le cours du récit, du présent puis du futur - verse dans l'onirisme en associant Éros et Thanatos. Les lièvres condamnés à mort par leur désir qui les expose aux tirs, clôturent le chapitre comme une possible projection métaphorique de leur chasseur improvisé, devenu anonyme, Julián lui-même qui se dérobe en cette fin de roman à son désir tu, au dilemme d'un amour contraire à sa vocation. L'animal ici n'est plus le chien, mais le monde de la chasse livre une nouvelle possibilité, la métaphore onirique des audaces que le personnage s'interdit de par son statut de prêtre, une lecture fantasmée de sa sexualité interdite.

Ce bref parcours à travers un classique du roman espagnol du XIX ${ }^{e}$ siècle pourra bien paraître anecdotique et centré sur des points mineurs, à l'exception de la séquence fameuse des chiens dans la cuisine. Il nous semble néanmoins révéler tout le profit qu'il y a pour le critique à se pencher sur le regard porté sur l'animal. La série de détails relevés - qui ne constituent pas même une thématique au sens traditionnel - surprend par la diversité des commentaires qu'ils suscitent: les chiens peuvent tour à tour 
intégrer une démonstration d'école du naturalisme, comme autant de signes d'un milieu et d'un mode de vie, et ressortir à un tout autre imaginaire littéraire, en jouant sur les fantasmes du loup ou du chien effrayant, lorsque le romanesque réactive une tentation fantastique ou gothique. Notre dernier exemple, non plus axé sur les chiens mais toujours sur la chasse, confirme ce sentiment que l'animal, même lorsqu'il n'est pourvoyeur que de détails pour la création littéraire, est associé à une puissante capacité de rêve et d'imaginaire. A ce moment où l'écriture s'écarte fortement de la manière du roman, Pardo Bazán semble portée par cette capacité qui lui procure un autre langage pour dire la sexualité dont son personnage est privé. Le détail animal, même dans un roman où les comportements et la psychologie humaine sont l'essentiel, ne peut manquer de se faire entendre.

\section{BIBLIOGRAPHIE}

ARASSE, Daniel (1996). Le détail, pour une histoire rapprochée de la peinture. Paris : Flammarion [1992]. CLARÍN, Leopoldo ALAS (2012). Cuentos morales, ed. Jean-François Botrel. Madrid : Cátedra [1896].

LANEYRIE-DAGEN, Nadeige (2016). Animaux cachés, animaux secrets. Paris : Citadelles et Mazenod.

MAUPASSANT, Guy de. (1974). Contes et nouvelles. Paris : Gallimard, Pléiade.

PARDO BAZÁN, Emilia (1997). Los Pazos de Ulloa. Madrid : Cátedra [1886].

ZAYAS, María de (1998). Desengaños amorosos. Madrid : Cátedra [1647].

\section{RÉSUMÉS}

Les figures canines, chiens de chasse familiers ou chiens-loups effrayants, s'avèrent autant de détails significatifs dans Los Pazos de Ulloa, roman de la ruralité galicienne qui marque en 1886 l'époque naturaliste de la romancière Emilia Pardo Bazán. Au début du roman, la promiscuité d'un enfant illégitime avec les chiens de chasse de son père, le marquis d'Ulloa, illustre de façon frappante la présentation du milieu dans la lignée de l'esthétique zolienne. Ce "pays de loups » est l'espace d'un déterminisme où l'interaction de l'homme et de l'animal est profonde. D'autres figures de chiens ou de loups génératrices d'angoisse correspondent à l'autre versant du roman, l'exploration psychologique et des souvenirs du roman gothique. La thématique canine opère ainsi comme un révélateur de la diversité du roman.

Dogs, either familiar retrievers or frightening wolfhounds, may appear as significant details in Emila Pardo Bazán's novel The Castle of Ulloa, a novel about the northwestern spanish rural world from her naturalist period. A well-known sequence, where a young child - the marquis of Ulloa's hidden child - walks on all fours among a pack of hounds, stands as a striking treatment of environment following Zola's aesthetics. The «land of wolves" around the manor is a playground for interaction between animals and men, here an element of naturalist determinism. Besides, other dog or wolf figures, generating fears, are part of another dimension 
of this novel, based on gothic recollections and psychological exploration. Animal details thus might be revealing of the diversity of inspiration.

INDEX

Mots-clés : animal , roman , naturalisme , Espagne, Bazán (Pardo)

Keywords : animal, novel, naturalism, Spain, Bazán (Pardo)

\section{AUTEUR}

YVES GERMAIN

Université Paris-Sorbonne, études ibériques

yg.germain[at]orange.fr 